\title{
Colloidal Nanostructures: In-Situ Electron Microscopy of Plasmon-Mediated Synthesis, Chemistry and Self-Assembly
}

\author{
E. Sutter ${ }^{1 *}$
}

1. Department of Mechanical and Materials Engineering, University of Nebraska-Lincoln, Lincoln, NE, USA.

* Corresponding author: esutter@unl.edu

Real-time transmission electron microscopy can be used to follow the behavior and measure the properties of nanostructures over a wide range of environmental conditions with resolution down to the atomic scale. Liquid-cell electron microscopy (LCEM), for instance, is the only technique that allows direct imaging of nanometer-scale processes in liquids. It has been successfully applied to imaging various processes in liquids, solutions, and colloidal suspensions that were typically investigated ex-situ on samples taken at different process stages, or in some cases in-situ using reciprocal space techniques.

Here we will illustrate the power of in-situ liquid-cell electron microscopy imaging to probe complex solution-phase processes in real space. Examples include plasmon-mediated colloidal synthesis of anisotropic nanostructures and the self-assembly of nanocrystal superstructures in solution.

Metallic nanostructures that carry localized surface plasmon resonances (LSPRs) have received particular attention due to their very strong visible-light coupling and ability to supply non-equilibrium charge carriers that can drive non-thermal processes by charge and/or energy transfer. We report in-situ experiments that provide unprecedented insight into plasmon-mediated materials synthesis [1]. We use the relativistic electron beam in transmission electron microscopy as a localized source of evanescent supercontinuum light to excite the LSPR of silver nanostructures while following their plasmonmediated growth with nanometer spatial resolution. Our observations show two distinct regimes: an early stage in which $\mathrm{Ag}$ nanoprisms grow almost exclusively laterally whilst keeping a constant thickness; and an advanced stage where, due to larger size and more complex plasmon mode structure, distinct local differences are seen in both thickening and lateral growth of the nanoprisms. Linking this heterogeneity to the distribution of plasmonic 'hot spots' allows us to quantify key elements of the plasmon-driven growth process, notably the range over which hot carriers can be transferred to drive chemistry.

Solution-phase self-assembly of nanocrystals into mesoscale structures is a promising strategy for creating functional materials from nanoscale building blocks. In-situ LCEM is the only technique that provides access to real-space dynamics, especially at small scale, and is capable of probing selfassembly processes in real space in the native liquid environment. We will show that LCEM can probe self-assembly of nanocrystals in solution from monomers to extended assemblies with nanometer scale resolution, and provide fundamental information on assembly pathways and forces for self-assembly of polyhedral nanoparticles [2] and highly anisotropic nanocrystals [3] controlled by non-specific interactions, and programmable crystallization of DNA-nanoparticle conjugates [4, 5] (Figure 1, below) and their real-time reconfiguration in response to external stimuli [6]. 
References:

[1] P Sutter et al., J Am Chem Soc 139 (2017), p. 6771.

[2] K Ouhenia-Ouadahi et al., Chem Mater 28 (2016), p. 4380.

[3] E Sutter et al., Nat Commun 7 (2016), p. 11213.

[4] P Sutter, B Zhang and E Sutter, Nanoscale 10 (2018), p. 12674.

[5] M Shekhirev, E Sutter and P Sutter, Adv Funct Mater (2019), p. 1806924.

[6] E Sutter et al., Nanoscale 11 (2019), p. 34.

[7] This material is based upon work supported by the U.S. Army Research Laboratory and the U.S. Army Research Office under grant number W911NF-17-1-0141. The authors acknowledge seed funding from the Nebraska Public Power District through the Nebraska Center for Energy Sciences Research.
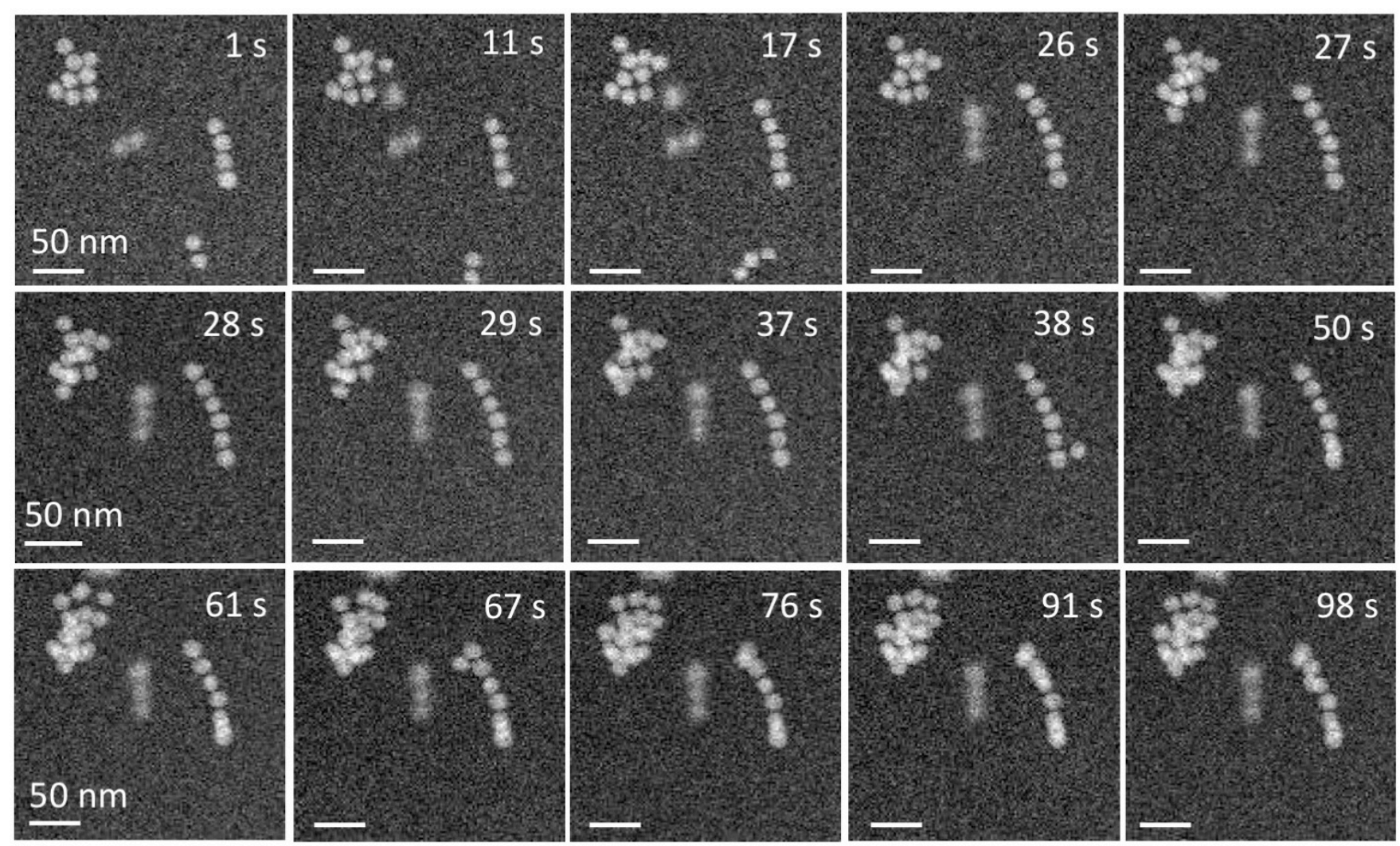

Figure 1. In-situ microscopy of the self-assembly of single strand (ss) DNA-Au nanoparticle conjugates into a compact 3D supracrystals and linear chains. The time-lapse image sequence shows selected HAADF-STEM images of the self-assembly of ssDNA-Au nanoparticle conjugates. Scale bars: $50 \mathrm{~nm}$. 